Cahiers de philosophie de l'université de

\title{
Remarques sur la mathématisation de la théorie du mouvement, entre réalisme et réflexivité
}

\section{Alain Michel}

\section{OpenEdition}

1 Journals

Édition électronique

URL : https://journals.openedition.org/cpuc/1356

DOI : 10.4000/cpuc.1356

ISSN : 2677-6529

Éditeur

Presses universitaires de Caen

\section{Édition imprimée}

Date de publication : 15 décembre 2008

Pagination : 161-191

ISBN : 978-2-84133-332-5

ISSN : $1282-6545$

\section{Référence électronique}

Alain Michel, « Remarques sur la mathématisation de la théorie du mouvement, entre réalisme et réflexivité », Cahiers de philosophie de l'université de Caen [En ligne], 45 | 2008, mis en ligne le 04 septembre 2020, consulté le 01 février 2023. URL : http://journals.openedition.org/cpuc/1356 ; DOI : https://doi.org/10.4000/cpuc.1356

\section{(c) (1) (5)}

Creative Commons - Attribution - Pas d'Utilisation Commerciale 4.0 International - CC BY-NC 4.0 https://creativecommons.org/licenses/by-nc/4.0/ 


\section{REMARQUES SUR LA MATHÉMATISATION DE LA THÉORIE DU MOUVEMENT, ENTRE RÉALISME ET RÉFLEXIVITÉ}

Dans ses Leçons sur la genèse des théories physiques, Jacques MerleauPonty affirme ${ }^{1}$ :

Malgré une tendance des mathématiciens à l'annexer, la Théorie physique est un discours mathématisé, mais non un discours mathématique, parce que la référence au monde réel et à l'expérience lui est essentielle... Malgré les mises en garde indéfiniment renouvelées et infiniment variées du "Positivisme», du «Pragmatisme", de l' Opérationnalisme", contre l'illusion d'un monde caché derrière les phénomènes, une visée ontologique réaliste est toujours présente dans la Théorie physique même sous ses formes les plus abstraites, et, à notre avis elle en est inséparable ${ }^{2}$.

Même si l'on est assez spontanément tenté d'adhérer à une telle position, il importe bien entendu de fournir des explications et des justifications. En quoi consiste le réalisme physique? De quelle nature est le réel posé par la théorie physique? En particulier, et c'est un point essentiel, le réalisme est-il susceptible de distinguer cette dernière de celles de la science voisine, sans laquelle il n'y aurait pas de loi, ni de théorie physique, les mathématiques? On a pu soutenir aussi une position réaliste en philosophie des mathématiques. Qu'est-ce qui distingue ces deux formes de réalisme? Il y a sur ces questions une littérature assez abondante, qui ne donne pas entièrement satisfaction en ce que, lorsqu'elle ne

1. Il nous semble que la thèse trouverait un appui solide dans la tradition de la pensée mécanique. Ainsi par exemple Cassirer souligne-t-il dans le même sens en commentant l'exposé des principes de la mécanique par Euler : «la signification objective des principes doit susciter l'exigence et l'hypothèse d'un terme de référence dans la réalité absolue. " Cf. Cassirer 1911, p. 482 sq.

2. Merleau-Ponty 1974, p. 9. 
demeure pas strictement philosophique, ce qui signifie d'un niveau de généralité peu satisfaisant du point de vue de la physique, elle s'occupe surtout des thèmes de la mécanique quantique. Elle tourne alors au commentaire des situations étranges ou paradoxales qu'on y rencontre, et donne lieu à des réflexions dont l'insuffisance provient essentiellement de leur caractère spéculatif et déraciné. L'analyse des positions philosophiques qui leur sert de préalable ne peut s'effectuer qu'après qu'on les a détachées de leurs sources réelles, en l'espèce de leurs sources physiques.

Nous tenterons une approche différente, fondée sur l'histoire.

Nous partirons de l'idée que, si la question du réalisme physique a un sens, nous ne pourrons le découvrir sans mobiliser les ressources de l'investigation historique, et qu'à tout le moins nous ne pourrons y accéder de manière complète que par l'histoire. La mathématisation de la théorie physique depuis Newton a rendu plus sensible un problème qu'elle n'a pas créé, celui de l'autonomie de la physique relativement aux mathématiques. On n'aurait pas de peine à le retrouver tout au long de l'histoire. Il suffirait de faire une revue rapide de quelques étapes fondamentales de l'histoire de la pensée physique, en commençant par le début, à savoir la physique d'Aristote, dans son mouvement critique du pythagorisme, en passant par Descartes et Leibniz, cartésien sur ce point, pour aller jusqu'à Kant. C'est en raison de cette volonté d'historiciser la réflexion que la thématique du mouvement, avec la question de son caractère absolu ou relatif, qui lui est essentiellement liée, me semble préférable à celle de la structure de la matière, objet principal de la théorie quantique, en même temps que de la plupart des analyses actuelles en matière d'épistémologie physique. Quelle est la réalité du mouvement, et de ce en quoi il a lieu, l'espace et le temps? C'est la question fondamentale, à l'aune de laquelle on peut évaluer les diverses théories du mouvement qui ont été proposées au cours de l'histoire. S'il est vrai que, pour reprendre les termes de Jacques Merleau-Ponty, la référence à une réalité est essentielle à la théorie physique en général, sa mathématisation, sous ses formes les plus puissantes, celles qu'on trouve dans le champ de la théorie de la relativité générale, ne saurait éliminer le problème du réalisme, mais seulement conduire à le poser à nouveaux frais.

C'est à cette question que les réflexions qui suivent sont consacrées, avec, à l'horizon, l'objectif d'inscrire la réflexion sur le réalisme physique dans le cadre plus large de l'histoire de la philosophie de la physique (Aristote, Descartes, Leibniz) et de la physique (Newton, Einstein, Mach, Poincaré). 
Un des premiers problèmes posés par la théorie de la relativité est "réflexif", c'est un problème de statut : appartient-elle à la physique ou aux mathématiques? Prenant acte du regain d'intérêt pour la Relativité Générale, laquelle avait subi la longue éclipse qu'on connaît, Andrej Trautman ${ }^{3}$ lui assigne plusieurs facteurs : manque de progrès importants de la physique théorique, espoir que sa combinaison avec la théorie quantique permettra de fonder la théorie des particules élémentaires, adoption comme modèle de théorie physique, essais pour transférer ses méthodes et concepts géométriques dans d'autres branches de la physique, tentatives pour relier la symétrie des particules élémentaires à la symétrie asymptotique des champs gravitationnels, etc. Il reconnaît aussi les difficultés qu'elle rencontre, en dépit de son accord avec l'expérience - corroboré par des résultats récents - à se faire accepter comme théorie physique. Il souligne qu'à l'heure actuelle, il s'agit de la meilleure des théories existantes de l'espace, du temps et de la gravitation, en même temps que d'un exemple assez exceptionnel de théorie bien fondée logiquement, dont la seule faiblesse consiste dans la minceur de sa confirmation expérimentale ${ }^{4}$. Quant à sa relation aux mathématiques, Hermann Weyl soulignait déjà ce fait que son influence a été dans tous les cas profonde, mais de nature bien différente selon qu'on l'entend sous l'une ou l'autre de ses deux formes. La restreinte a eu l'influence la plus forte sur la physique; ce fut le contraire pour la relativité générale, qui a eu l'influence la plus forte sur les mathématiques, de plus faible portée sur la théorie physique.

\section{La fonction réflexive de la mathématisation physique}

La mathématique, sous la forme que nous lui connaissons aujourd'hui, est une discipline qu'on peut qualifier de fondamentalement

3. Trautman 1973, p. 179-201; Trautman 1966a, p. 319-336; Eisenstaedt 1986, p. 115-185.

4. On a pu lui contester le titre de "théorie physique». S'il demeure entendu qu'on peut toujours restreindre la signification du mot "physique» à des théories de forte confirmation expérimentale, il paraît déraisonnable de le faire au point de devoir éliminer la plupart des travaux actuellement produits des recherches physiques effectives. Or, s'il est vrai que la théorie était initialement pauvre en résultats expérimentaux, la situation a changé au cours de la deuxième moitié du $\mathrm{XX}^{\mathrm{e}}$ siècle, à la suite d'importantes découvertes dans le domaine de l'astrophysique (pulsar), des mesures radio et radar (développement de la radioastronomie, usage de télescopes ultra-terrestres) de la détection des ondes gravitationnelles, etc. (Cf. Trautman 1973, p. 179). 
réflexive, au sens où elle ne cesse d'engendrer et de systématiser, à l'intérieur du corps de connaissance qu'elle constitue, des propositions qui portent sur elle-même en tant que connaissance - des thèses qui constituent la réponse à des problèmes qui sont plutôt des méta-problèmes, des problèmes du second ordre. À la différence des autres, ces derniers portent sur la mathématique, beaucoup plus qu'ils ne proviennent $d e$, ou apparaissent dans, la mathématique. On peut considérer cette forme de réflexivité, en dépit du caractère historiquement tardif de sa mise en lumière, comme une propriété d'essence des mathématiques. Elle les individualise en tout cas dans l'ensemble des autres sciences. Dans aucune autre science, on ne trouve, semble-t-il, ce niveau d'une connaissance proprement réflexive, dans lequel une discipline traite les métaénoncés, les énoncés qui portent sur la discipline en tant que discipline, comme les énoncés de la discipline eux-mêmes, avec les mêmes outils et les mêmes méthodes. Cette élimination de la séparation entre la discipline et sa matière, son thème ou sujet, ne se retrouve pas en effet sous cette forme visible dans les autres sciences. C'est une banalité de faire remarquer que dans les autres sciences, on distingue entre phénomènes et lois des phénomènes. Ainsi, en physique, distingue-t-on parfaitement entre la chaleur et les lois de la chaleur. En mathématiques, les lois, et donc la théorie, des objets, sont elles-mêmes objets - par exemple les lois de certains objets, envisagées en et pour elles-mêmes, donnent ce nouvel objet qu'est le groupe, ou l'espace, ou la topologie, ou tout cela à la fois, car on peut considérer aussi les objets d'un groupe comme liés par des lois de structure topologique, etc. ${ }^{5}$. On pourrait donc s'entendre pour distinguer par un tel critère la mathématique de la physique, même mathématique, s'il est vrai que celleci ne peut, en tant que discipline, se constituer comme thème de la recherche physique. L'objet de la recherche physique est, jusqu'à preuve du contraire, le monde extérieur, lequel n'inclut pas, sauf thèse réaliste extrême, de partie d'elle-même qui soit constituée de théorie ou de concept.

L'introduction, par Hermann Weyl et Elie Cartan, dans la théorie de la Relativité Générale, et dans un cadre de géométrie différentielle (riemanienne), du concept de connexion a abouti à

5. C'est le spectacle, familier dans la mathématique d'aujourd'hui, qui pour certains, est sans doute déjà d'hier, des emboîtements d'ensembles, hiérarchies des structures, superpositions des catégories - jusqu'au sommet où l'on trouve l'ensemble des ensembles, la catégorie des catégories... 
un traitement qui rend possible son exposition comme théorie de certaines structures - structures de variétés paramétrisées par un groupe, puis d'espaces fibrés. L'intérêt de cet investissement structural de la théorie est mathématiquement indéniable. Il est aussi, plus paradoxalement, philosophique et historique. Il a permis en particulier, ainsi qu'Élie Cartan en a fourni la brillante démonstration, un exposé de la mécanique newtonienne comme théorie d'une certaine variété d'espace-temps, et même d'un certain espace fibré. Cette présentation ne fournit pas seulement, par application rétrospective, un outil précis d'évaluation, ou de comparaison historique, des diverses constructions théoriques de l'espace et du temps chez Galilée, Leibniz et Newton. Elle donne aussi directement le passage à la théorie de la Relativité Générale, qui apparaît, si l'on adopte ce point de vue, comme une généralisation directe et naturelle, sinon tout à fait immédiate, de la mécanique newtonienne - ce que Cartan veut signifier lorsqu'il parle à ce propos de "simple changement de langage" ${ }^{6}$. Le mouvement commencé alors a été poursuivi par un certain nombre de théoriciens de la Relativité Générale, il a abouti à ce que l'on peut considérer comme une mise en forme structurale, qui constitue, semble-t-il, une bonne illustration de ce mouvement de réflexion intérieur à la théorie qui nous est apparu comme étant le propre de la mathématique.

Prenons l'exemple de la mécanique newtonienne. On peut décrire ainsi la structure géométrique de l'univers newtonien.

a) L'univers, ou espace-temps, soit $W$, est l'ensemble des événements (spatio-temporels) ordonné par la relation temporelle $" x$ antérieur à $y$ ", qui fournit directement la relation de simultanéité : "aucun (événement) $x$ n'est antérieur à $y$ ", qui est une relation d'équivalence. Le quotient de $W$ par cette dernière relation est «le temps». On appelle "instant» toute classe d'événements par la relation de simultanéité : le temps $T$ est l'ensemble de tous les instants. On peut introduire sur $T$ une notion de rapport d'intervalles permettant de le caractériser comme espace affine réel 1-dimensionnel, ou ligne euclidienne.

Cette structure affine est compatible avec l'ordre défini sur $W$.

Pour tout instant, on définit une notion de rapport de distances sur les événements appartenant à l'instant. Il est possible d'en donner une définition intrinsèque. Toute classe d'événements simultanés devient alors une partie d'un espace euclidien à trois dimensions,

6. Cartan 1923, p. 327 ; Cartan 1984, p. 661. 
y acquérant une structure euclidienne, et la réunion de ces «espaces instantanés", ou "espaces de simultanéité", forme le lieu de tous les événements possibles, qu'on appelle "espace-temps".

b) Il s'agit d'obtenir, à partir de là, un cadre de référence pour l'analyse du mouvement. Les espaces instantanés 3-dimensionnels qu'on vient de définir sont entièrement séparés. On va définir, pour les relier entre eux, une "connexion cinématique» de l'espace-temps. L'idée est de les insérer dans une structure plus large d'espace affine réel à quatre dimensions, dans laquelle on définit une notion de «ligne droite» pouvant servir à représenter tous les mouvements rectilignes uniformes possibles. La projection de $W$ sur $T$ est une "application affine", et, naturellement, les structures euclidiennes des espaces instantanés sont compatibles avec leurs structures affines, en tant que fibres de cette application. L'espace-temps peut alors être défini comme une variété affine à quatre dimensions avec: une variété affine réelle à une dimension, qui représente le temps ; une projection (affine) de la première sur la seconde : projection "temporelle", qui assigne à tout événement possible sa "date»; sur chaque fibre de la projection, une métrique euclidienne compatible avec la structure affine.

Si $\mathbf{E}$ est un espace euclidien 3-dimensionnel, et $\mathbf{T}$ un espace 1dimensionnel affine, on obtient pour l'espace-temps une structure de produit direct:

$$
W=\mathbf{E} \times \mathbf{T}=\{\langle s, t\rangle ; s \in \mathbf{E}, t \in \mathbf{T}\}
$$

c) Pour définir, analogiquement à la date, le «lieu » de tout événement possible, il faut introduire une projection spatiale, projection dont les fibres forment une famille privilégiée de lignes droites parallèles, qui sont les "axes du temps». Inversement, si on sélectionne une direction d'espace-temps transversale aux espaces instantanés, on obtient une projection bien définie sur l'espace à tout instant, projection qui a des fibres parallèles à cette direction. Le fait que l'espace-temps n'ait pas d'axe du temps privilégié constitue le "principe de relativité de Galilée ».

Dans $\mathbf{E} \times \mathbf{T}$, on a quelque chose comme un «espace de permanence». Pour tout point de l'espace, la fibre de la projection spatiale au-dessus de ce point peut être considérée comme "le point, persistant dans le temps". Ainsi l'état de repos absolu, dont la possibilité caractérise l'espace newtonien se définit par une fibration. On notera que l'application de la relativité galiléenne cache cette structure : en éliminant l'axe du temps, elle garde la projection sur $\mathbf{T}$, mais non celle sur $\mathbf{E}$. Il y a bien entendu un groupe de transfor- 
mations qui préserve la structure de l'espace-temps newtonien: c'est le produit direct du groupe des dilatations/rotations/translations de $\mathbf{E}$ par le groupe affine de $\mathbf{T}$, que Weyl a appelé "groupe élémentaire». C'est un groupe de Lie à neuf dimensions.

On voit qu'on a en fait tous les éléments d'un espace fibré.

Cette première présentation appelle deux remarques.

D'abord on obtient ainsi une formulation géométrique, donc précise, des absolus newtoniens. Pour deux événements quelconques $p, q$, on considère qu'on peut objectivement décider s'ils sont simultanés et s'ils ont lieu au même endroit. L'espace et le temps sont absolus au sens précis où, pour toute paire de points de $W$, il y a une séparation spatiale et une séparation temporelle, toutes les deux définies de manière unique. C'est là le point essentiel, et non pas la structure topologique ou métrique de $\mathbf{E}$ et de $\mathbf{T}$, qu'on peut prendre par exemple comme étant celle de $\mathbf{R}^{3}$ et de $\mathbf{R}$ et qui, pourvu que la condition ci-dessus soit réalisée, pourrait fort bien être différente.

On note ensuite que la stratification de $W$ engendrée par les parties maximales d'événements simultanés peut être interprétée comme la structure causale de W. L'hyperplan $t=t(e)$ qui passe par un événement $e$ sépare le futur causal (ou domaine d'influence) de $e$ de son passé causal ${ }^{7}$.

Dans un deuxième temps, on se propose de développer la géométrie de l'espace-temps dans un cadre suffisamment général pour

7. La circonstance que le futur et le passé de $e$ ont une frontière commune, le "présent", exprime l'hypothèse, implicite en physique newtonienne, qu'il y a des signaux arbitrairement rapides, réalisables par le moyen de corps rigides ou d'une action instantanée à distance. La stratification obtenue de l'espace-temps est la contrepartie formelle d'une ontologie de l'évolution du monde extérieur dans le temps. L'état présent du monde est considéré comme consistant dans la distribution de matière dans l'hyperplan existant "maintenant " et la succession des configurations des corps dans les hyperplans représente l'histoire de l'univers matériel, cela non seulement pour un observateur donné, mais objectivement, en soi, en quelque sorte. Dans une telle présentation, l'espace-temps de Leibniz a moins de structure que celui de Newton. En fait, en adoptant comme norme la structure qu'on vient de définir, on pourrait dire qu'il n'a pas assez de structure, alors que celui de Newton en a trop. Il n'a notamment ni mouvements, ni axes spatiaux non rotatifs privilégiés. Il n'a pas de structure affine : la notion de parallélisme pour des quadri-vecteurs n'y a pas de signification. Des deux espaces temps de Newton et de Leibniz, on peut dire qu'ils sont adéquats dans leur critique réciproque, inadéquats dans leurs positions respectives. Du point de vue actuel, il leur manque, au-delà des structures causale et métrique, une structure affine, dont les droites temporelles sont les mouvements libres (sur tout cela, voir Ehlers 1975, p. 73-75). 
accueillir les extensions ultérieures, notamment relativistes. Il faut introduire des coordonnées. On a alors besoin de la structure de variété, et surtout de celle de variété différentiable.

Soit toujours $W$ une variété différentiable à quatre dimensions (quadri-dimensionnelle), homéomorphe à $\mathbf{R}^{4}$, donnée par un système de coordonnées $\left\{x^{\mathrm{i}}\right\}, i=1, \ldots, 4$, global. On introduit sur $W$ deux structures géométriques supplémentaires :

(1) un recouvrement de $W$ par une famille à un paramètre d'hypersurfaces $t=t\left(x^{i}\right)$ avec la topologie de $\mathbf{R}^{3}$ : ce seront les "espaces instantanés" (de simultanéité absolue).

(2) on montre qu'il existe un champ vectoriel $u$ ("gradient de vecteurs ") engendré par les normales aux espaces instantanés, satisfaisant partout

$$
\sum_{1 \leq i \leq 4} u_{i} n_{i}=1
$$

les $n_{i}=\frac{\partial t}{\partial x^{i}}$

étant les composantes de la normale, soit $n$, à la surface ( $t=$ constante) au point en question.

Les trajectoires de ce champ forment une "congruence" (ou "famille congruente") à 3 -paramètres de courbes: $\lambda^{\alpha}=\lambda^{\alpha}\left(x^{i}\right), \alpha=$ $1,2,3$.

Toute courbe coupe un espace instantané ( $t=$ constante) en un point et un seul. Ces courbes sont les «lignes temporelles» de l'espace absolu. On peut obtenir ainsi des espaces instantanés : $x^{4}=c t e$, et une congruence de courbes de la forme $x^{\alpha}=$ cte.

Dans une telle structure, on ne peut pas avoir de métrique d'espace-temps non singulière. Cela signifie qu'on a toujours deux métriques différentes, lesquelles sont associées à deux structures géométriques. Il y a séparation des métriques : l'une définit l'intervalle temporel entre deux points, l'autre définit la distance spatiale entre deux points sur les courbes ${ }^{8}$.

8. Si les deux points sont $x^{i}$ et $x^{i}+d x^{i}$, la distance spatiale est :

$$
\sqrt{\sum_{1 \leq i \leq 4} n_{i} n_{j} d x^{i} d x^{j}}=\sqrt{\sum_{1 \leq i \leq 4} \frac{\partial t}{\partial x_{i}} \frac{\partial t}{\partial x_{j}} d x^{i} d x^{j}} ;
$$

si les deux courbes sont $\lambda^{\alpha}, \lambda^{\alpha}+d \lambda^{\alpha}$, la distance temporelle est : $\sqrt{\delta_{\alpha}^{\beta} d \lambda^{\alpha} d \lambda^{\beta}}$ ( $\delta$ est le symbole de Kronecker; pour deux points du système de coordonnées particulier donné ci-dessus, la distance spatiale est fournie par la formule euclidienne ordinaire). 
On peut obtenir, à partir de là, un espace-temps plus large et général que l'espace-temps newtonien, et qu'on appelle, selon les auteurs, "quasi-newtonien " ou "galiléen». Il a des propriétés originales et intéressantes par rapport à l'espace-temps newtonien. En particulier, on ne peut y parler de positions et de vitesse absolues ${ }^{9}$.

Il s'agit maintenant de relier entre eux ces espaces instantanés. C'est ce que rend possible la procédure suivante, dite de "connexion".

On peut définir un produit scalaire sur les espaces tangents à la variété espace-temps, ce qui permet de récupérer la géométrie euclidienne sur les espaces tangents, et d'effectuer la liaison requise, en s'inspirant des idées de Levi-Civita et Cartan ${ }^{10}$. Pour effectuer ce raccordement, et réaliser la connexion entre le temps et l'espace qui n'est plus possible par une métrique, on postule ce qu'on appelle une "connexion affine" dite "plate" ou "affinité plate",

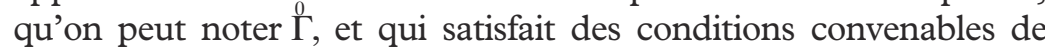
"constance covariante" (ou constance pour la "différentiation covariante"). La longueur des vecteurs spatiaux, ou tangents à un espace instantané, notamment, est conservée dans la différentiation covariante.

L'affinité définit ainsi une notion moins forte que la relation de congruence, celle de parallélisme. La ligne qui correspond à la géodésique est ici le "chemin le plus droit", c'est-à-dire une ligne (courbe) qui reste "parallèle à elle-même", au sens où le vecteur tangent en tout point reste parallèle à lui-même - c'est l'idée même de «transport parallèle» de Levi Civita. L'équation en est (pour un

9. Dans ce dernier, il n'y a pas de séparation spatiale en général. La distance temporelle entre deux points, ou événements, quelconques est invariante (il y a un temps absolu), mais la distance spatiale entre deux points n'est définie que pour des points situés dans le même plan de simultanéité - donc seulement si les points appartiennent à des sous-espaces, à des "coupes tri-dimensionnelles" simultanées. Cela implique qu'on ne peut parler ni de position ni de vitesse absolues (invariantes) contrairement à ce que pensait Newton; il y a bien un tenseur contravariant de rang 3 tel que $g^{i j} n_{j}=0$, mais, étant de rang 3 , donc ininterprétable comme tenseur métrique d'espace-temps, s'il peut jouer le rôle d'un tenseur métrique pour les espaces instantanés d'espace-temps, il ne peut le faire pour l'espace-temps lui-même.

10. Élie Cartan a reconnu lui-même qu'il était redevable à Levi Civita de la notion de transport parallèle (on dit aussi "translation "), dont il a fait une pièce mâ̂tresse, après généralisation, de sa théorie des espaces "à connexion". Il s'agit d'un déplacement qui laisse invariant le produit scalaire (défini, dans un espace riemannien, sur les espaces tangents), et en particulier les longueurs et les angles des vecteurs. Voir par exemple Cartan 1924, p. 294-320 ; Cartan 1984, p. 863889 . 
système de coordonnées quelconque $z^{i}, t$ étant ici un paramètre arbitraire le long de la ligne) :

$$
\frac{d^{2} z^{i}}{d t^{2}}+\Gamma_{j k}^{i} \frac{d z^{j}}{d t} \frac{d z^{k}}{d t}=0
$$

En résumé, on a deux hypothèses fondamentales:

(1) La première pose l'espace-temps comme une variété différentiable quadri-dimensionnelle, continue, et cela semble vrai même dans les théories unifiées du champ qui postulent un plus grand nombre de dimensions, comme celles de Kaluza-Klein ${ }^{11}$. L'espace-temps n'y est jamais qu'un quotient quadri-dimensionnel d'un espace "plus gros" par une relation d'équivalence. Cela signifie que l'espace est toujours considéré comme un continu. Même dans les théories qui tentent d'opérer ce qu'on appelle une "quantisation» de la Relativité Générale, on constate que le point de départ est une variété différentielle. Ce fait pourrait conduire à dire qu'on a là le principe le plus fondamental de la physique, un principe commun à toutes les théories physiques présentées jusqu'à maintenant. Ce principe est souvent considéré comme si évident qu'il paraît inutile d'y insister. Il est pourtant digne d'intérêt, et Eintein a considéré avec le plus grand sérieux le problème de savoir pourquoi nous considérons naturellement l'espace et le temps comme formant un continu ${ }^{12}$.

(2) La seconde pose l'existence d'une connexion affine (qu'on suppose ordinairement symétrique) dans l'espace-temps. Les géodésiques de cette connexion forment une classe privilégiée de lignes d'univers de la théorie considérée. La nature de cette connexion peut varier selon la théorie considérée, ou selon la solution particulière de cette théorie, mais l'existence même d'une connexion affine demeure comme un trait commun à toutes ces théories. Sa justification est profonde, à la fois structurelle (elle est liée au caractère local des lois) et historique (elle a son origine dans la formation même de la dynamique). Elle est nécessaire pour que les lois fondamentales de la physique puissent être exprimées sous la forme

11. Les travaux de Theodor Kaluza (en 1921) et d'Oskar Klein (en 1926) sont considérés comme pionniers pour la théorie unifiée des champs. Inspirée de suggestions de Gunnar Nordström et Hermann Weyl, leur idée était de porter le nombre de dimensions de l'espace-temps à 5 . On trouve une tentative du même type chez Jordan et Thiry; cf. Trautman, 1966b, p. 413-414.

12. On a peu de théories alternatives, posant par exemple un espace-temps comme une structure discrète, représenté par un treillis régulier de points. Il y a eu semble-t-il une tentative de Bohm en ce sens, qui n'a pas été jugée satisfaisante. 
d'équations différentielles, et une telle exigence vaut pour toutes les théories physiques.

On voit aussi qu'il s'agit toujours de géométrisation physique, et non de géométrie pure. La géométrisation d'une théorie comme celle du mouvement implique la position d'hypothèses mécaniques correspondantes. Il convient ensuite, évidemment, de déterminer par des expérimentations physiques tous les objets qu'on a pu construire par des moyens mathématiques, ce qui exige une méthode de détermination physique de la connexion. Il faut chercher un ensemble privilégié de courbes dans la variété, susceptibles d'être interprétées comme les géodésiques de la connexion affine, et donc chercher un ensemble privilégié de courbes d'univers de l'espacetemps qui puisse recevoir une telle interprétation.

La première question à poser à une théorie physique est donc celle-ci. Quelle est la classe privilégiée de lignes d'univers qu'on doit interpréter comme ensemble des géodésiques de la connexion affine de la variété d'espace-temps?

Prenons d'abord la mécanique newtonienne.

On y distingue classiquement deux cas, selon qu'on y inclut ou non la gravitation.

\section{Premier cas, en l'absence de gravitation}

L'hypothèse supplémentaire de l'existence d'une grandeur scalaire $t$ (appelée "temps absolu») telle que les hypersurfaces $t=$ constante soient des 3-espaces euclidiens signifie bien que dans toute hypersurface de ce type (dans tout espace : $t=$ constante), il y a un système privilégié de coordonnées cartésiennes, mais elle ne dit rien sur la connexion entre des systèmes pour différentes valeurs de $t$.

C'est le rôle de la première loi de la dynamique que d'établir une telle connexion. Elle fournit la classe privilégiée de lignes d'univers. C'est sans doute la loi la plus importante de la physique théorique. On peut la décomposer en deux parties :

(a) il existe une classe privilégiée de mouvements de corps appelés «mouvements» libres (inertiels) qui sont les mouvements des corps sur lesquels n'agit aucune force.

(b) il existe un système de coordonnées global $\left\{x^{\alpha}\right\}, x^{4}$ étant le temps, dans lequel les mouvements libres sont caractérisés par les équations :

$$
\left(d^{2} x^{\mathrm{a}} / d t^{2}\right)=0 \quad \alpha=1, \ldots, 4
$$

Cette dernière égalité montre que les trajectoires des mouvements libres peuvent être considérées comme les géodésiques de la 
connexion affine dont les composantes s'annulent dans ce système de coordonnées particulier, ce qui permet de formuler la première loi de Newton de manière géométrique ${ }^{13}$. Le tenseur de courbure de la connexion affine s'annule. Une telle connexion affine est dite «intégrable». Les systèmes de coordonnées vérifiant (1) sont dits « inertiels». Ils sont caractérisés par l'annulation des composantes de la connexion affine relativement à eux. Ils sont déterminés à une transformation linéaire arbitraire près des $x^{\alpha}$ qui préservent $x^{4}$ comme un temps absolu, c'est-à-dire invariants par les transformations linéaires de la forme :

$$
\begin{aligned}
& x^{\alpha} \rightarrow A_{\beta}^{\alpha} x^{\beta}+c^{\alpha} t+d^{\alpha} \\
& t \rightarrow a t+b
\end{aligned}
$$

où $A_{\beta}^{\alpha}, a, b, c, d$ sont des constantes réelles, $a \neq 0$ et la matrice $A_{\beta}^{\alpha}$ est non singulière (transformations qui forment un groupe).

Une telle présentation est applicable aussi à la théorie de la Relativité Restreinte.

\section{Deuxième cas, en présence de gravitation}

La première loi de Newton ne peut être postulée sous la forme cidessus, puisqu'il ne peut y avoir de mouvements libres, c'est-à-dire de corps sur lesquels aucune force ne s'exerce. La gravitation est en effet une force de portée infinie qui ne peut être éliminée par aucun moyen connu, et les expériences montrent que, pour tous les corps, la masse est proportionnelle à la charge gravitationnelle. C'est le principe même de l'égalité ou équivalence de la masse inerte et de la masse pesante. Le mieux est alors de considérer la chute libre des particules, c'est-à-dire le mouvement dans un champ gravitationnel d'où toutes les interactions sont éliminées, donc d'adopter, comme classe privilégiée de mouvements, les "chutes libres", ou trajectoires de corps soumis à la seule force de gravitation. La première loi ainsi modifiée prend la forme suivante, toujours essentiellement double :

- il existe une classe privilégiée de mouvements de corps appelée "chutes libres", suivies par les corps soumis à la seule force de gravitation.

13. Pour la mécanique newtonienne stricto sensu, on peut se restreindre à trois composantes, et écrire $d^{2} x / d t^{2}=d^{2} y / d t^{2}=d z^{2} / d t^{2}=0$, c'est-à-dire prendre $d x_{a} / d t^{2}, a=1$, 2, 3. La première loi se formule alors de la manière suivante : le mouvement d'une particule correspond à une ligne d'univers dans l'espace-temps. C'est une courbe coupant l'hypersurface $t=$ constante en un point et un seul. 
- il existe un système de coordonnées global et une fonction réelle $\phi$ telle que les chutes libres soient caractérisées par l'équation: $\left(d^{2} x^{\alpha} / d t^{2}\right)=\left(-\partial \phi / \partial x^{\alpha}\right)^{14}$.

En fin de compte, ces deux descriptions fournissent chacune une structure différente: une structure inertielle, constituée par les trajectoires libres, qui est une notion dynamique; une structure affine, constituée par les chemins les plus droits, qui est une notion géométrique. Il s'agit de deux structures conceptuellement distinctes, mais qui coïncident dans l'espace-temps quadri-dimensionnel quasi-newtonien. On a donc déjà une géométrisation de l'inertie, même en l'absence même d'une métrique spatio-temporelle.

On remarquera enfin que, dans ces structures, les principes d'invariance ou de relativité fonctionnent comme de véritables énoncés réflexifs.

Un principe d'invariance apparaît comme un méta-énoncé, un "énoncé sur", plutôt qu'un "énoncé de»: c'est un énoncé qui porte sur un énoncé - ou un ensemble d'énoncés - physique(s).

Soit $E$ un énoncé physique quelconque. Désignons notre principe d'invariance par « $\mathrm{R}(E)$ ", il s'énoncera par exemple :

si $E$ est une loi de la nature, alors $E$ est invariant (ou covariant).

14. En fait, il existe dans l'espace-temps une connexion affine telle que les lignes d'univers des particules en chute libre sont des géodésiques relativement à elle. Les particules en chute libre déterminent la connexion affine dans l'espacetemps. Les coefficients de la connexion ont la forme :

$$
\Gamma_{\mu \nu}^{\lambda}=\stackrel{0}{\Gamma}_{\mu \nu}^{\lambda}+h^{\lambda \kappa} \frac{\partial \phi}{\partial x^{\kappa}} t_{\mu} t_{v}
$$

où $\Gamma$ est la connexion affine plate, $t_{k}=\partial t / \partial x_{k}$, $t$ étant le temps absolu, $h^{\lambda \kappa}$ est le tenseur métrique de l'espace euclidien $t=$ cte (il est constant au sens covariant relativement à $\Gamma), \phi$ est la fonction potentiel, $h^{\lambda \kappa}=\left(\nabla x^{\lambda}\right)\left(\nabla x^{\kappa}\right)$, où le symbole $\nabla$ dénote le gradient $\nabla=(\partial / \partial \mathrm{x}, \partial / \partial \mathrm{y}, \partial / \partial \mathrm{z})$. La grandeur $t$ peut être normalisée de manière à devenir un paramètre affine le long des géodésiques. Si on le fait, l'équation d'une géodésique prend la forme d'une loi newtonienne de mouvement dans un champ gravitationnel :

$$
\frac{d^{2} x^{\lambda}}{d t^{2}}+\stackrel{0}{\Gamma}_{\mu \nu}^{\lambda} \frac{d x^{\mu}}{d t} \frac{d x^{v}}{d t}=-h^{\lambda \kappa} \frac{\partial \varphi}{\partial x^{\kappa}} .
$$

Le terme en $\Gamma$ correspond aux forces qui se manifestent quand on utilise des systèmes de coordonnées non inertiels. Le référentiel peut toujours être choisi tel qu'on ait $x^{0}=t, x^{1}=x, x^{2}=y, x^{3}=z$, et

$$
\stackrel{0}{\Gamma}_{\mu \nu}^{\lambda}=0 \text {. }
$$

L'équation du mouvement prend alors la forme usuelle : $d \mathbf{r}^{2} / d t^{2}=-\nabla \phi$. 
Il faut évidemment établir cet énoncé qui porte sur l'énoncé " $E$ », ce qui ne peut se faire que dans la théorie physique dont il relève. Nous dirons que la loi physique est exprimée par une certaine proposition dans une théorie physique (langage) et que le principe d'invariance énonce qu'une certaine autre proposition, exprimant l'invariance de la première, est démontrable dans la théorie physique en question, ce qui signifie à partir de ses axiomes mathématiques. Il devient alors un méta-énoncé, un énoncé qui dit quelque chose sur une théorie. On peut donc dire qu'en physique théorique, un "principe" est un énoncé sur des théories physiques, fondé sur des expériences ou énoncé par extrapolation à partir de théories connues. Et, en général, un principe a une signification pour toute une classe de théories physiques, celles pour lesquelles il est vrai, et à l'égard desquelles il joue un rôle de sélection.

Examinons de ce point de vue le cas des deux grands principes de la théorie de la relativité.

\section{Le principe dit "de relativité restreinte»}

Il représente une classe de théories supposant un espace affine plat comme modèle d'espace-temps. Du point de vue dynamique, ces théories décrivent les mouvements libres des particules ponctuelles au moyen d'une famille de droites dans l'espace affine, famille qui forme un sous-ensemble ouvert non vide de l'espace des droites. Chacune de ces théories est caractérisée par une structure nondynamique additionnelle: connexion linéaire intégrable, tenseur métrique (en mécanique newtonienne et en Relativité Restreinte), temps absolu (en mécanique newtonienne), éther (comme système d'inertie privilégié, absolument au repos en tout point de l'espace), etc. À chaque théorie est associé un groupe d'automorphismes approprié. Il s'agit en général d'un groupe de transformations affines conservant la structure additionnelle d'espace-temps qui a été introduite. Ici, c'est-à-dire pour la Relativité Restreinte, il s'agit des transformations opérant transitivement dans la famille des mouvements libres.

\section{Le principe dit "de relativité générale»}

Pour en donner une formulation précise, on peut utiliser la distinction proposée par Trautman entre deux sortes de grandeurs physiques: des grandeurs absolues, ou externes, d'une part, les variables dynamiques d'autre part. Ces dernières satisfont seules les équations du mouvement et correspondent à des degrés de liberté du 
système. Elles caractérisent l'histoire d'un système physique, les éléments absents étant les mêmes pour toutes les histoires. Par exemple, le tenseur métrique est un élément absolu dans la Relativité Restreinte, mais devient un élément dynamique dans la Relativité Générale ${ }^{15}$. Une symétrie, ou automorphisme, du système, qui, physiquement, signifie une mise en correspondance de tout état de mouvement physiquement admissible du système avec un autre état du même système ${ }^{16}$, est une transformation qui conserve ou laisse invariantes les grandeurs absolues.

Le principe peut alors être énoncé ainsi : les automorphismes ou symétries d'une théorie sont les transformations qui préservent l'absolu. Dans la théorie relativiste de la gravitation, le groupe d'automorphismes consiste dans l'ensemble des difféomorphismes d'espace-temps. En général, il représente la classe de théories supposant un espace affine courbe comme modèle d'espace-temps, associée à un groupe d'automorphismes qui sont des difféomorphismes d'espace-temps ${ }^{17}$. L'invariance est alors déterminée comme covariance. Il s'agit d'un principe fort, distinct de la simple possibilité de changer de coordonnées curvilignes.

Dans cette voie de la mathématisation structurale, on a pu aller jusqu'à émettre l'idée de l'organisation possible des théories, ou des modèles mathématiques de théories, dans des catégories au sens strict, celui de la théorie mathématique des catégories. Les objets de la catégorie galiléenne, Gal, par exemple, seraient les espaces galiléens, espaces affines dotés d'une application bilinéaire symétrique positive de rang $n-1$ (si $n$ est la dimension de l'espace vectoriel associé), sur lesquels on peut définir des morphismes affines

15. L'identification des grandeurs comme externes ou dynamiques dépend, dans la plupart des cas du problème considéré et des approximations à effectuer. Par exemple, pour le problème newtonien à un corps, les grandeurs absolues sont les éléments géométriques de l'espace-temps et le potentiel. Pour le problème à deux corps, ce sont les éléments qui se réfèrent à l'espace-temps : le groupe de symétrie est plus grand et inclut, outre les rotations, les déplacements et les transformations galiléennes.

16. Ou transformation qui laisse invariante l'équation du mouvement. Plus précisément, si on considère, dans le système $\mathrm{E}$ de tous les états, le sous-système F de ceux qui peuvent être effectivement réalisés, déterminés par les lois du mouvement, une symétrie est une correspondance 1-1 de E dans E qui applique $\mathrm{F}$ dans $\mathrm{F}$.

17. Trautman (1966a, p. 322) en donne une autre caractérisation intéressante. À l'intérieur d'une théorie, les lois physiques ne peuvent contenir que les variables dynamiques et les éléments absolus appropriés à cette théorie. 
particuliers, dits "morphismes galiléens». Les «transformations galiléennes" sont des automorphismes de ces espaces ${ }^{18}$.

La mobilisation des concepts de base de la théorie des fibrés et de catégories comme ceux de produit fibré et de fibré trivial (pour la première), de transformation naturelle (pour la seconde) permet d'éclairer les enjeux fondamentaux des grandes théories du mouvement depuis Galilée. La généralité du langage ou du traitement structural permet à la fois de dégager les analogies, c'est sa fonction unificatrice, et de faire apparaître avec précision les différences à l'origine des théories, c'est sa fonction de sélection physique. En mécanique newtonienne, par exemple, l'espace-temps est représentable comme produit du temps par l'espace, mais on n'en a aucune représentation "naturelle" au sens où on pourrait exprimer cette représentation en termes des transformations qu'on appelle "naturelles» en théorie des catégories. Si l'on adopte ce critère, on ne peut dire en toute rigueur, comme plus haut, que l'espace-temps de la mécanique newtonienne est un produit. Ce fait pourrait signifier que l'espace y reste relatif. Il n'y a pas de méthode absolue pour établir si deux événements simultanés se produisent au même endroit ou non. Il n'y a pas de découpage naturel de l'espace - alors même qu'il demeure un découpage naturel pour le temps. On pourrait en trouver une confirmation dans l'existence, à l'intérieur de la catégorie Gal, d'un foncteur covariant de Gal dans le fibré $(\mathrm{E}, \mathrm{T}, \pi)$, qui n'est pas naturellement équivalent au foncteur de Gal dans celle des produits fibrés.

On obtiendrait un résultat analogue avec la Relativité Générale, qu'on peut comparer de ce point de vue à la Relativité Restreinte. On peut prendre ici comme catégorie sous-jacente celle des variétés différentiables avec connexion linéaire (une procédure de transport parallèle), soit LDiff.

En Relativité Restreinte, il n'y a pas de fibration naturelle sur le temps, donc l'espace-temps n'est pas un produit, mais, à cause de l'existence d'une connexion linéaire intégrable par transport parallèle, le fibré des repères est trivial: c'est un produit.

18. D'autres exemples seraient fournis, en mathématiques par la catégorie des espaces affines, notée le plus souvent Aff, celle des fibrés différentiels (fibrés sur variétés différentielles), $D F i b r$, celle des fibrés principaux, PFibr; en physique, par celle des espaces de phase, $P h$, celle des champs de vecteurs, Vfibr, celle des fibrés vectoriels sur des variétés différentiables à $\mathrm{n}$ dimensions, ou encore celle des espaces hilbertiens complexes en physique quantique, notée Hilb (voir Trautman 1975). 
En Relativité Générale, la non-intégrabilité de la connexion linéaire (le fait que le résultat du transport parallèle le long d'une courbe dépend de la courbe) pourrait se manifester dans la théorie par la non-trivialité du fibré des repères. Ce dernier est trivial pourvu seulement que la variable E n'ait pas une topologie noneuclidienne.

À ce niveau de la géométrisation, il n'y a donc pas de différence entre les deux théories de la relativité. Dans les deux cas, le fibré des repères est trivial. Pour faire apparaître la différence, il faut précisément faire intervenir la théorie des catégories et sa notion de naturalité. La différence réapparaît au niveau du concept d'équivalence naturelle de catégories.

Sur la catégorie des espaces affines, qui convient à la fois pour la physique galiléenne et la Relativité Restreinte, on peut définir deux foncteurs de la catégorie des fibrés principaux. Le premier va de $E$ sur le fibré des repères $B(E)$, le second, de l'espace affine $(E$, $\mathrm{V},+$ ) sur le produit de $\mathrm{E}$ par le fibré des repères. Ils sont naturellement équivalents. La signification physique de ce fait mathématique est qu'un système de coordonnées global sur $\mathrm{E}$ induit un isomorphisme du fibré des repères $B(E)$ sur le produit $E \times G L(n)$.

En revanche, dans la catégorie des variétés différentielles, il n'y a pas d'équivalence naturelle entre foncteurs sur le fibré des repères et foncteurs sur les fibrés produits.

En résumé si l'on prend les énoncés sur les espaces qui sont ou non des produits comme se référant à l'équivalence naturelle de foncteurs appropriés, on obtient deux énoncés simples :

(1) le principe de relativité de la Relativité Restreinte (affirmant que le groupe des automorphismes opère sur la famille des mouvements libres) implique que l'espace-temps E n'est pas un produit.

(2) le principe de relativité de la Relativité Générale, ou covariance (affirmant que le groupe des automorphismes, difféomorphismes de l'espace-temps, opère transitivement sur la famille des chutes libres), implique que le fibré des repères $B(E)$ n'est pas un produit.

Trautman ${ }^{19}$ donne le récapitulatif suivant (E est l'espace-temps):

19. Ibid., note précédente, p. 190. Une connexion linéaire induit une champ privilégié de bases linéaires sur $\mathrm{B}(\mathrm{E})$ et transforme ainsi $\mathrm{B}(\mathrm{B}(\mathrm{E}))$ en un produit. Du coup, on peut se demander s'il existe une théorie d'espace-temps où $B(B(E))$ n'est pas un produit..., ce qui revient à se projeter hardiment dans le futur de la physique. 
- espace-temps prégaliléen ${ }^{20}: \mathrm{E}$ est le produit cartésien de l'espace par le temps (absolus) $\mathrm{E}=\mathrm{T} \times \mathrm{S}$,

- espace-temps galiléen: E est un fibré sur la base T (temps),

- espace-temps minkowskien (de la Relativité Restreinte) : E n'a pas de fibration naturelle, mais le fibré des repères $B(E)$ est un produit (fibré),

- espace-temps einsteinien (de la Relativité Générale) : le fibré des repères $B(E)$ n'est pas un produit, mais admet un "transport parallèle" qui transforme $\mathrm{B}(\mathrm{B}(\mathrm{E}))$ en un produit (fibré).

Il nous semble qu'on a ici un exemple particulièrement frappant de la puissance des structures mathématiques à l'œuvre. Non seulement les structures, en particulier les fibrés, nous procurent un point de vue profond sur la structure de l'espace-temps et la nature des théories, mais elles induisent de véritables méta-raisonnements : les énoncés peuvent être de même structure, sans porter sur les mêmes espaces. Nous proposons d'interpréter ce type de résultats comme le signe que la théorie physique est entrée, à la suite de la mathématique, dans le régime de la réflexivité.

\section{La question du réalisme}

\section{Le problème du réel dans la théorie physique contemporaine}

Le devenir de la microphysique est là pour nous rappeler qu'à l'origine de la théorie physique se trouve l'effort de compréhension de ce qui se passe réellement dans le monde naturel. Un siècle après la découverte du noyau atomique, nous n'avons, au sujet des forces nucléaires, que des lambeaux d'explication, et, en ce qui concerne les particules élémentaires, que des théories formelles et purement classificatoires, dont la valeur explicative est très mince, relativement au luxe de néologismes et d'images dont s'encombre leur vocabulaire ${ }^{21}$. Il est devenu progressivement évident que les

20. Ou en électrodynamique prérelativiste.

21. L'idée que les véritables besoins de la physique concernent les idées et non l'accumulation d'expériences, avait déjà été nettement articulée par Einstein dans une correspondance à Besso : "seule une spéculation hardie est à même de nous faire progresser. Du matériel empirique incompréhensible, nous en avons plus qu'il n'en faut..." (cité par Cornelius Lanczos, cf. Lanczos 1974). 
difficultés rencontrées par la théorie avaient un caractère fondamental. Liées, aux yeux de certains, à l'absence de progrès théorique essentiel dans les autres branches de la physique théorique, elles apparaissent lors du regain d'intérêt suscité par la Relativité Générale durant ces dernières décennies, depuis 1960 environ. L'espoir naît alors, chez certains physiciens, qu'en combinant la Relativité Générale avec la théorie quantique, on parvienne à donner un fondement à la théorie des particules élémentaires et même à construire une théorie unifiée, géométrique, de l'espace, du temps, et de la gravitation. Beaucoup de physiciens considèrent qu'il y a un lien direct entre la crise de la cosmologie quantique et les premières grandes discussions sur les fondements de la mécanique.

Il semble qu'il y ait deux grands problèmes.

Le premier vient de l'incompatibilité apparente entre la Relativité Générale et les principes de la mécanique quantique. La dynamique newtonienne n'était pas incompatible avec l'électro-magnétisme de Faraday et Maxwell. Par un procédé dit "de quantisation", les deux théories sont susceptibles de passer d'une forme classique à une forme quantique. Mais les essais pour appliquer le même procédé à la Relativité Générale et pour créer la "gravité quantique» ont jusqu'à présent échoué ${ }^{22}$.

Le second problème consiste dans la relation entre la physique quantique et la physique classique. Il semble que la physique quantique soit plus fondamentale que la physique classique, et qu'elle s'applique à de plus grands objets, voire à l'univers entier. Il pourrait y avoir une théorie quantique de l'univers, c'est ce qu'on appelle la "cosmologie quantique». Mais c'est un fait que la physique quantique n'existe pas sous une telle forme. Une partie de celle-ci décrit le comportement effectif des atomes, molécules et radiations, une autre partie consiste dans des règles étranges qui opèrent à l'interface des mondes microscopiques et macroscopiques. De fait, l'exemple même d'un univers apparemment unique est un véritable puzzle à l'intérieur de la mécanique quantique.

$\mathrm{Au}$ total, la situation est donc très insatisfaisante. Elle ébranle la croyance, qui reste profonde chez beaucoup de physiciens, en l'unité de la nature. Parce que la Relativité Générale est en même temps une théorie de la gravité et de la structure à grande échelle de l'univers, la création de la cosmologie quantique exigera

22. Voir les travaux de Dirac et d'autres, et la mise en évidence des problèmes concernant l'interprétation du temps. 
certainement la solution du problème (qui n'est guère plus étroit) de la "gravité quantique».

Certains auteurs se sont demandé si ce chiasme ne viendrait pas, pour une grande part, des idées peu critiques, bien que profondément ancrées, que se font les physiciens de la nature de l'espace, du temps et des choses. Par exemple, une hypothèse que les physiciens font couramment et spontanément consiste à poser l'existence d'une multiplicité de choses en mouvement dans un cadre invisible d'espace et de temps. Les discussions qui ont eu lieu sur les fondements de la Relativité Générale portent sur ces questions fondamentales. Qu'est-ce que le mouvement? Qu'est-ce que l'espace? Qu'est-ce que le temps? Quels sont les principes qui doivent guider la théorie du mouvement? Les théories existantes du mouvement, qu'il s'agisse de la mécanique newtonienne ou de la relativité générale, sont en excellent accord avec l'observation. Les doutes à leur égard procèdent donc plutôt de considérations philosophiques générales que d'une inadéquation pratique des prédictions, et l'approche exigée est théorique : qu'est-ce qui est réel?

La tâche est critique, de critique philosophique et historique. Sur chacune des questions évoquées plus haut, on constate que des points de vue radicalement différents se sont fait jour au cours de l'histoire, avant Einstein, et du temps même de Newton. L'œuvre physique d'Einstein est un monument. Il convient, selon nous, de se montrer attentif à ce que la reconnaissance de son importance n'aboutisse pas à faire obstacle à l'interrogation sur la nature et l'origine réelle du cadre d'espace-temps, à empêcher la réflexion sur les principes, à bloquer l'exercice de la régression historique, libératrice de différentes possibilités théoriques.

Avant Einstein ou de son temps même, dans des travaux dont on est sûr qu'Einstein les a connus, des physiciens comme Carl Neumann, Ernst Mach, Henri Poincaré avaient pris conscience de l'exigence de nouvelles fondations pour la physique. Les écrits de ce dernier notamment, au début du $\mathrm{XX}^{\mathrm{e}}$ siècle, contiennent des suggestions assez claires sur la manière dont on aurait pu développer et faire émerger de la dynamique, prise comme thème principal des recherches, une théorie explicite de l'origine réelle du cadre spatio-temporel.

\section{L'analyse dynamique : une difficulté fondamentale}

Pour donner un aperçu plus précis des problèmes ainsi soulevés, il peut être utile de partir de ces difficultés de la mécanique newto- 
nienne soulignées par Mach et par Poincaré. Il semble qu'on ne saurait les tenir pour résolues du seul fait de l'existence de la théorie de la Relativité Générale.

Il y a ainsi, dans la mécanique newtonienne, une lacune fondamentale que Poincaré a soulignée de manière particulièrement remarquable ${ }^{23}$. La dynamique prétend pouvoir prédire le futur d'un système physique à une condition : que les positions et vitesses initiales de tous les corps dans ce système soient connues (avec leurs masses, charges, etc., et les lois de force). Étant données ces positions et vitesses, l'évolution future du système est en effet déterminée de manière unique par les lois de Newton. Mais Poincaré souligne avec vigueur que ces données ne le sont en fait que dans l'espace absolu. L'unicité de la prédiction n'est vraie que si les vitesses initiales sont connues dans l'espace absolu, disons, en termes modernes, dans un référentiel d'inertie. Ayant souligné ce fait, il se demande ce qui arrive si on connaît seulement les données intrinsèquement observables, c'est-à-dire les distances relatives initiales, et la vitesse initiale à laquelle ces distances relatives changent.

Reprenons, pour fixer les idées, un modèle de représentation proposé par Barbour ${ }^{24}$. Considérons un univers consistant en $\mathrm{N}$ points matériels en mouvement dans un espace euclidien à deux dimensions, selon les lois de Newton. Imaginons que l'on puisse par un moyen quelconque (au moyen par exemple de photographies) fixer sur des feuilles d'épreuves les configurations des corps dans l'univers au fur et à mesure qu'il passe par ces configurations successives. Le champ de la dynamique newtonienne peut être représenté par cette pile de feuilles successives transparentes disposées l'une sur l'autre avec des intervalles de séparation de longueur constante (représentant des unités égales et successives de temps). Si le concept newtonien d'espace absolu a un sens, chaque configuration ayant une position définie dans l'espace absolu, il devrait être possible de placer chaque configuration (correspondant à un instant) dans une position déterminée sur la feuille correspondante. Or, supposant qu'on nous donne les feuilles et qu'on nous demande de les ranger de cette manière, on se rend compte immédiatement qu'on ne peut pas appliquer la prescription newtonienne, parce que les feuilles n'ont pas de trait caractéristique. Si on considère chacune

23. Voir surtout Poincaré 1902, chapitre V. Poincaré se livre à la même analyse critique à propos de temps dans Poincaré 1905, ch. II.

24. Cf. Barbour 1982, p. 251-274. Nous lui devons une grande partie des remarques qui suivent. 
des feuilles, il n'y a rien qui puisse nous conduire à la placer sur la feuille correspondante. À un instant donné, la vieille objection de Leibniz contre l'espace absolu paraît irréfutable.

Mais si on suppose donné l'ensemble des feuilles, connues les masses et charges des corps, et garanti le fait qu'elles suivent les lois de Newton, alors, en principe, les feuilles pourront être empilées. En pratique, on peut même s'arranger pour que trois feuilles suffisent à déterminer un rangement, pourvu que nous sachions qu'elles sont séparées par de courts intervalles de temps donnés ${ }^{25}$. La construction est presque unique, au sens suivant. La configuration initiale peut être placée dans une position arbitraire : toutes les configurations successives tournent alors exactement de la même manière. L'espace absolu empiriquement construit tourne comme un tout, d'un bloc, et cette rotation n'affecte pas les relations internes, intrinsèquement observables, entre les corps. C'est pourquoi l'argument initial de Leibniz contre l'espace absolu échoue à réfuter le mouvement absolu. Le centre de masse du système pris comme un tout peut avoir une vitesse uniforme arbitraire, mais cette dernière est facile à éliminer, puisque l'hypothèse que le centre de masse reste fixe n'affecte pas les relations internes entre les $\operatorname{corps}^{26}$.

Le "futur» ne peut être "prédit» de manière unique que si on a un empilement déterminé des deux premières feuilles. Si on donne les deux premières configurations et si on les place sur les deux feuilles, on peut en principe en déduire toutes les autres. Mais si on donne seulement les deux configurations sans déterminer l'emplacement initial des deux photos l'une relativement à l'autre, il est tout

25. Par exemple, si on dispose de quelques corps neutres, on sait qu'ils suivent nécessairement des lignes droites, selon la première loi. Pour empiler les feuilles, on doit donc seulement s'assurer que ces quelques corps neutres, choisis comme corps-tests, satisfont la première loi. Une fois les feuilles empilées au moyen des corps neutres, les positions et vitesses initiales des corps restants peuvent se déduire des deux premières lois, et on peut les utiliser pour calculer les accélérations, donc les positions que ces corps devraient avoir dans la troisième feuille. On peut alors vérifier la théorie en comparant avec les positions actuelles. Une réalité précise peut être ainsi assignée à l'espace absolu.

26. Une des grandes faiblesses, aux yeux d'un moderne, de l'argumentation de Leibniz est le fait qu'il raisonne en termes d'une seule configuration. Un tel raisonnement est rarement pertinent en dynamique parce que, dans le schéma ci-dessus, un emplacement différent de la première feuille engendrera seulement une translation et une rotation de la pile entière : les relations internes, observables, ne seront pas affectées. 
à fait impossible de prédire le futur de manière univoque. Cela, parce que, à partir de deux feuilles, il est impossible de déduire le moment angulaire du système. Ce dernier est engendré par la manière dont les deux feuilles sont placées l'une relativement à l'autre, et non par les configurations intrinsèques qu'elles représentent.

Comment la critique de Poincaré se formulerait-elle dans ce cadre?

Dans la construction ci-dessus, la considération des données intrinsèquement observables que réclame Poincaré correspond à la donnée de deux feuilles seulement de la succession (à partir desquelles les vitesses initiales peuvent être construites avec une précision infinitésimale). Dans ce cas, la mécanique newtonienne ne peut pas prédire le futur de manière univoque. La difficulté est que les données relatives ne donnent pas d'information du tout sur la rotation générale du système. Quand les astronomes considèrent un système tel que le système solaire, donc un sous-système du système global, l'état de rotation est déduit à partir d'une référence à une variété extérieure au système, par exemple les étoiles distantes, laquelle procure la pièce manquante d'information exigée pour déterminer l'évolution unique des données initiales (nous concevons intuitivement que le système solaire a réellement un moment angulaire relativement à l'univers comme tout). Qu'arrive-t-il quand on considère l'univers entier? Il n'y pas alors de variété extérieure à laquelle on puisse faire appel. Il semble que, dans ce cas, des données initiales identiques (dans tous leurs attributs perceptibles) puissent conduire à des évolutions dynamiques entièrement différentes du système. L'évolution future peut être complètement différente parce qu'elle dépend du moment angulaire effectif réalisé dans le système. La raison profonde s'en trouve dans le fait que la mécanique newtonienne est décrite par des équations différentielles du second ordre temporel - lui-même évidemment en relation avec le fait que, d'un point de vue dynamique, les grandeurs décisives sont les accélérations ${ }^{27}$.

27. Cela est parfaitement contraire au principe d'identité des indiscernables, qui fondait déjà l'objection de Leibniz à l'espace absolu de Newton. On peut donc caractériser la mécanique newtonienne par son conflit avec le principe d'identité des indiscernables. Comme le remarque Barbour (1982), on pourrait dire en schématisant que le principe d'identité des indiscernables est appliqué à un champ différent par Leibniz, Poincaré, Mach. Alors que Leibniz l'applique à une seule configuration, Mach le fait au mouvement pris comme un tout, et Poincaré aux seules données initiales. Du point de vue de Mach, si l'univers entier était accessible à l'observation, la distinction newtonienne ne serait pas 


\section{Les limites de l'analyse dynamique d'Einstein}

Une des lacunes de l'édifice laissé par Einstein consiste dans le fait qu'il n'a pas clairement montré en quoi consistaient les principes philosophiques permettant de considérer une théorie vraiment relativiste prise en un sens strict, à la manière de Mach et Poincaré, de la gravitation comme souhaitable et réalisable ${ }^{28}$.

En 1905, en raison de ses doutes à leur égard, Einstein évite les modèles dynamiques explicites. Il remplace l'espace et le temps newtoniens par l'entité nommée "espace-temps" et construit la réalité physique au moyen d'énoncés portant sur le comportement des règles et des horloges dans l'espace-temps. Mais ce comportement n'est jamais que postulé, il n'est pas engendré à partir de la structure de l'espace-temps. En choisissant de procéder ainsi, Einstein a assuré ses fondations physiques, mais il n'a pu le faire qu'en prenant le risque de s'éloigner de ses vues intuitives primordiales sur la nature de l'espace et du temps. Peut-on les retrouver en revenant aux points de vue qui furent ceux de Mach et de Poincaré?

Reprenons l'exemple fondamental du principe de relativité.

On sait que traditionnellement, il a revêtu deux formes différentes: celle du principe dynamique de Galilée, énonçant qu'il existe une classe de repères distingués, ceux en mouvement relatif uniforme, qui sont équivalents pour l'expression des lois de la nature; celle du principe de relativité cinématique, plus primitif et moins spécifique, qui affirme seulement l'inaccessibilité à la vue,

illusoire. Bien qu'on ne puisse guère nier que le mouvement soit relatif en tant qu'observé, il semble qu'il y ait néanmoins un sens dans lequel le mouvement relatif observable révèle une origine absolue. Tout se passe donc comme si on avait besoin d'un "principe de Mach ». C'est ce qu'a dû ressentir Einstein. On pourrait aussi invoquer un principe de raison suffisante. Supposons que nous nous trouvions nous-même dans un univers dans lequel les lois de Newton s'appliquent et que, de plus, l'univers comme tout ait un moment angulaire non nul. On peut alors demander: quelle est la raison pour que l'univers ait ce moment angulaire particulier ? On ne peut en donner aucune qui soit exprimable en termes d'entités authentiquement observables.

28. Einstein a passé plusieurs années à construire une théorie qui satisfasse le "principe de Mach». On connaît ses hésitations : il croit d'abord avoir réussi avec la Relativité Générale, puis il doute. Il reprend les équations du champ. Il finit par abandonner le principe. Il a vraisemblablement cru que les développements de la physique, en particulier de l'électromagnétisme de Faraday et Maxwell, avec le concept de champ, rendaient la mécanique de Mach obsolète. Les difficultés soulevées par Mach à ce niveau lui ont paru insurmontables. 
et à l'observation en général, de l'espace et du temps. L'espace et le temps sont invisibles, inobservables. Tout ce que nous voyons, ce sont des objets en mouvement relatif. Si on ne peut parler du mouvement et de la position d'un objet que si l'on dit combien cet objet est éloigné des autres objets, la position et le mouvement sont relatifs aux autres objets. Les deux principes sont d'une importance capitale pour la mécanique, la théorie du mouvement, de l'espace et du temps. Les deux occupent une place centrale dans l'histoire de la physique ${ }^{29}$.

Comment penser leur relation? Il semble qu'une manière de rendre justice à Poincaré à ce propos serait de dire qu'il a voulu unifier les deux principes en un seul, posant une unique condition. Celle-ci aurait porté sur la structure de la dynamique. La relativité dynamique est depuis Galilée un fait physique, dont a pris acte Newton, en l'incorporant à une mécanique qui était par ailleurs absolutiste. C'est l'enjeu du corollaire 5 des Principia..., qui, sans menacer la cohérence interne de l'édifice, ouvre des questions difficiles relatives à la signification profonde de la mécanique newtonienne. Héritier du mouvement de pensée critique qui s'était fait jour dans la deuxième moitié du XIX ${ }^{\mathrm{e}}$ siècle, notamment chez Helmholtz et Mach, eux-mêmes dans la descendance de Riemann et de Kant, Poincaré ne pouvait se satisfaire d'une telle solution. Il en a fait apparaître, dans des analyses célèbres, mais dont on n'a peut-être pas toujours suffisamment dégagé la force, le caractère de compromis instable. Il est possible qu'il ait eu le projet de dériver le principe d'un critère naturel de relativité cinématique. Mais c'était en 1902, avant que la théorie relativiste d'Einstein ne réalise entre l'espace et le temps une intrication telle qu'elle rendait désormais difficile de savoir si une telle dérivation eût été possible.

Le problème apparaît dans toute sa force dès qu'on se demande comment les référentiels distingués, qui jouent un rôle vital dans la Relativité Restreinte, sont pratiquement déterminés. Quelle est leur origine? On ne peut l'assigner sans faire intervenir la matière observable, et donc le principe de relativité au sens cinématique. Ces questions sont assez négligées par les physiciens, qui les jugent sans doute trop philosophiques, et ni Einstein ni Minkowski n’y ont répondu, n'ont même vraiment cherché à y répondre.

29. Ce serait donc une erreur de présenter l'histoire de manière strictement linéaire, en faisant comme si Galilée avait substitué le premier, dynamique, au second, cinématique. Ce dernier, utilisé comme on sait, au Moyen Âge, l'est encore par Copernic, Descartes, et Galilée lui-même... 
Einstein ne s'est guère intéressé à la détermination pratique des repères distingués. Il les prend simplement pour acquis. Les repères sont approximativement réalisés sur la terre par des machineries soigneusement mises au point. Mais comment les trouvonsnous dans l'espace? Ce n'est pas une question triviale. Mais ce n'est pas une question pour laquelle il semble manifester un intérêt quelconque, pratique ou théorique. Einstein se demande ce que sont les lois de la nature dans des repères donnés. Il ne se demande pas s'il y a des lois qui déterminent les repères eux-mêmes. Au mieux, à une telle question, il n'a cherché qu'une réponse indirecte ${ }^{30}$. Autant qu'on puisse en juger, il concevait l'espace-temps comme physiquement réel, formant un contenant réel pour ces entités matérielles qu'on nomme champs et particules. Un tel mode de pensée appartient à une tradition allemande qui a sa source la plus nette, en ce qui concerne l'espace, chez Riemann ${ }^{31}$. Sans doute Einstein reconnaissait-il que les points de l'espace-temps, étant invisibles, ne pouvaient être distingués et identifiés que par la matière observable présente en eux. Mais, comme l'espace-temps était rendu visible par une telle matière, il supposait aussi fondamentalement qu'il pouvait y insérer des lignes de coordonnées susceptibles de servir de moyen d'expression pour les lois de la nature. Si tel est bien le cas, et le point apparaît décisif, il suscite ce qui est sans doute la plus grande des lignes de fracture qui séparent sa pensée de celle de Poincaré. Rien dans la réalité physique ne suggère que les lignes de coordonnées devraient être tracées de telle manière plutôt que de telle autre. Il n'y a pas de normativité physique des référentiels. Tout choix est arbitraire et viole le principe de raison suffisante.

30. En un sens Einstein les a abolis, puisque, dans l'espace-temps de la relativité générale, le réel est fait de courbure. Le réseau des lignes de coordonnées fictives est extérieur à son support. En Relativité Générale, les lignes de coordonnées ne sont au fond que des marques, ou des inscriptions, sur une réalité sous-jacente, et les coordonnées elles-mêmes que des noms par lesquels on désigne et on identifie les points de l'espace-temps. On retrouve la question de la conception einsteinienne de la structure de ce dernier.

31. Nous pensons bien sûr à son mémoire d'habilitation (Habilitationsvortrag) Über die Hypothesen, welche der Geometrie zu Grunde liegen soutenu le 10 juin 1854 devant un jury dans lequel siégeait Gauss, et qui ne fut publié qu'en 1866. Riemann y utilise l'expression Raum au sens d'espace physique, une notion qu'il interprète comme instance du concept général d'espace compris comme grandeur plusieurs fois étendue (mehrfach ausgedehnte Grösse). La géométrie est cette branche de la connaissance qui traite spécifiquement de l'espace physique, par particularisation, ou exemplification, du concept général de relations métriques. 
Pour Einstein, il y avait dans cette situation quelque chose d'intolérable. La très forte conviction de la rationalité de la nature le conduisait inéluctablement à penser que la seule solution satisfaisante était la théorie posant la possibilité d'exprimer les lois de la nature dans tous les systèmes existants sous la même forme, c'est-à-dire la théorie de la Relativité Générale ${ }^{32}$. Quoiqu'il dise par ailleurs, notamment quand il prétend que la condition de covariance générale prive l'espace-temps de son dernier vestige de stabilité physi$q_{\text {que }}^{33}$, Einstein traite l'espace comme une réalité directement accessible, quelque chose d'aussi réel qu'un objet concret, sauf l'invisibilité. D'un point de vue conceptuel, il n'a pas d'objection à considérer l'espace-temps comme une variété existante, physiquement réelle, à laquelle on peut attacher, au moins en pensée, ces marques que sont les coordonnées. Pour rendre l'idée épistémologiquement acceptable, il devait supposer que l'espace-temps est, de quelque façon, "rendu visible» par la présence, en lui, de ces marques matérielles - élevées alors au statut de référentiels ${ }^{34}$.

Comme ce fut assez souvent le cas dans l'histoire, pour des raisons sans doute diverses, ni les objections issues de l'idéalisme mathématisant de Poincaré, ni celles venues de l'empirisme sensationniste de Mach n'ont pu durablement contrarier le déploiement de la "visée ontologique réaliste" que J. Merleau-Ponty voyait à l'œuvre au cœur de la théorie physique. Elle est plus que jamais présente dans la réflexion einsteinienne, et le succès final de la

32. Mach, comme Poincaré, considère que l'entité «espace» n'a pas d'existence réelle. Il ne faut pas oublier qu'un de ses objectifs est l'élimination des concepts métaphysiques, inobservables, auxquels il convient de substituer, en toute circonstance, des relations démontrables entre objets directement observables. L'espace fait partie de tels concepts.

33. C'est apparemment de Sitter qui a montré en quoi consistait le véritable enjeu : la covariance générale prive les coordonnées d'espace-temps de toute signification physique directe. L'important, selon de Sitter, est qu'Einstein ait aperçu l'inadéquation de la représentation en coordonnées.

34. Voir à ce propos les textes amusants dans lesquels Einstein évoque, pour rendre la chose plus imagée, des "mollusques de référence ». Par exemple en 1917 (cf. Einstein 1917) : «on utilisera des corps de référence non rigides... qui pourraient être appelés pratiquement des mollusques de référence... Tout point du mollusque doit être traité comme un point d'espace." Du temps même d'Einstein, un physicien comme Minkowski posait manifestement l'existence réelle de l'espace-temps. Einstein était trop pénétré des conceptions de Mach pour ne pas en douter. Mais il continue à utilliser l'espace comme un simple intermédiaire, support de conditions et rien d'autre. Pour lui, tout se passe apparemment comme si le support avait une consistance propre susceptible de lui conférer un rôle indépendant. 
théorie de la Relativité Générale, en dépit des incertitudes qui la marquent dès l'origine et ne cessent ensuite de l'accompagner, a été une fois de plus celui du réalisme physique.

\section{Conclusion}

La théorie de la Relativité Générale, a dit un de ses promoteurs récents, comporte deux aspects, qui se partagent également les faveurs des physiciens: l'un mathématique, l'autre physique. De manière homologue, il est légitime de distinguer, dans le mouvement de sa genèse, la mathématisation d'une part, la conceptualisation physique d'autre part.

Il semble que les exemples traités autorisent une conclusion dans ce double registre.

Les mathématiques ne se contentent pas de fournir à la physique un langage de rigueur et de précision pour la formulation des vues intuitives et des résultats expérimentaux. Elles lui procurent aussi, par la vertu de la généralisation, des modèles d'explication. Dans le devenir récent de la théorie de la Relativité Générale, la contribution mathématique franchit une nouvelle étape. La mathématisation engage la théorie physique dans un mouvement de réflexivité, irréversible et, autant qu'on puisse en juger, indéfini. Les exemples évoqués d'incorporation à la théorie des fibrés et des catégories, me paraissent ici les plus significatifs. On aura remarqué qu'ils tournent autour de la notion, fondamentale en physique théorique, mais d'essence mathématique, puisqu'il s'agit toujours d'un groupe, d'invariance.

Supposons qu'il soit possible de réduire toutes les formes d'invariances physiques, ou invariances ordinaires des lois physiques, à un concept unique, débarrassé de toute condition spécifique caractéristique des théories particulières, comme lorsqu'on applique la méthode géométrique pour rendre une théorie «indépendante des coordonnées". On obtiendrait une sorte d'invariance canonique. En l'état actuel des choses, ce serait, disons, la covariance. On peut chercher à caractériser cette dernière comme une espèce d'équivalence, au sens mathématique, entre plusieurs formulations de théories physiques déjà invariantes, c'est-à-dire à l'origine desquelles on a construit une condition d'invariance. La notion d'équivalence naturelle, au sens de la théorie des catégories, permet alors de distinguer, et de déployer dans leur profondeur, ces théories. Deux espèces de structures, par exemple de variétés, étant données, la cova- 
riance consiste dans la possibilité de leur équivalence, équivalence qui permet à son tour de les représenter comme des formalisations d'une seule et même théorie physique. Leurs degrés d'invariance, supposés comparables au sens où des groupes d'invariance sont reliés entre eux par inclusion, restent différents. Le moteur d'une telle ascension dans la généralité et l'abstraction ${ }^{35}$, à laquelle il n'est guère possible d'assigner un terme, ne nous semble pas autre chose que la forme prise, dans la théorie physique, par le mouvement que nous avons appelé "réflexivité». Réduites à leur noyau invariant, les théories sont prises à leur tour comme thème d'une étude mathématique dont les outils sont fournis par les structures.

On voit bien que, dans un tel mouvement, la théorie physique gagne rigueur et précision, mais court en même temps le risque de perdre son autonomie en devenant partie pure et simple des mathématiques - de mathématiques elles-mêmes happées par l'ascension formelle. Et pourtant, dans un champ comme celui de la mécanique, qui concerne le mouvement de corps physiquement existants, voire matériels, la mathématisation ne saurait épuiser les ressources de l'analyse. Aussi puissants que soient ses résultats, elle ne saurait se substituer à la conceptualisation physique. Cette dernière reste guide indispensable. C'est en ce sens, nous semble-t-il, que Jacques Merleau-Ponty a bien raison de souligner, comme il le fait dans la citation donnée au début, que la référence à la réalité reste essentielle à la théorie physique. En matière d'analyse du mouvement, c'est encore le recours à l'histoire qui permet de le montrer avec le plus d'acuité. L'histoire montre les progrès d'une réflexion toujours plus attentive à saisir la réalité physique. C'est bien de l'origine et de la nature réelle de l'espace, du temps, du mouvement en général, puis de l'inertie, de la relativité, etc., que débattent, même si c'est quelquefois à distance temporelle, des penseurs de la physique comme Mach, Poincaré, Einstein, voire Galilée ou Newton. Il est dans le pouvoir de la réflexion épistémologique de le montrer, de préciser ce qui est en jeu dans les interrogations et les multiples argumentations et discussions qui les entourent. Peut-elle aller audelà ? Lui appartient-il de prendre parti quant aux positions respectives qui résultent de ce qu'on pourrait appeler les jugements physiques de réalité, de trancher par exemple entre Newton et Leibniz, ou entre Einstein d'un côté, Mach ou Poincaré de l'autre, ou encore,

35. Voir notamment les études de Sneed 1971, et Scheibe 1978; cf. Agassi et Cohen 1981. 
pour nous limiter à une des alternatives rencontrées par l'analyse la plus commune, entre la position d'une causalité physique absolue et l'exigence d'observabilité empirique? Nous ne le croyons pas. Le travail de l'histoire, philosophiquement pratiquée, peut contribuer à éclairer la réflexion en identifiant les héritages, en montrant les contraintes, en préparant les nécessaires ruptures. Il ne saurait d'aucune façon se substituer au travail du physicien. S'agissant de théorie physique, c'est à ce dernier qu'il revient, à ses risques et honneur, de montrer la voie de la vérité.

Alain MICHEL

Université d'Aix-Marseille 


\section{Références bibliographiques}

Agassi J. et CoHEN Robert S. (1981) (éd.), Scientific Philosophy Today, New York, Springer (Boston Studies in the Philosophy of Science; vol. 67).

BARbour J. (1982), "Relational Concepts of Space and Time», British Fournal of Philosophy and Science, 33, p. 251-274.

CARTAN É. (1923), "Sur les variétés à connexion affine et la théorie de la relativité généralisée", Annales scientifiques de l'École Normale Supérieure, 1, p. 325-412.

CARTAN É. (1924), "Les récentes généralisations de la notion d'espace», Bulletin des Sciences Mathématiques, 48, p. 294-320.

Cartan É. (1984), Euvres, Paris, Éditions du CNRS, t. III.

CASSIRER E. (1911), Das Erkenntnisproblem in der Philosophie und Wissenschaft, Berlin, Verlag Bruno Cassirer, 2 vol.

EISENSTAEDT J. (1986), "La relativité générale à l'étiage, 1925-1955", Archive of History of Exact Sciences, 35, p. 115-185.

EHLERS J. (1975), "The Nature and Structure of Spacetime», American Fournal of Physics, vol. 43, 2, The Physicist's Conception of Nature, J. Mehra (éd.).

EINSTEIN A. (1917), Über die Spezielle und die Allgemeine Relativitätstheorie, Gemeinverständlich (rééd. Vieweg \& Sohn, Braunschweig, 1920).

LANCZOs C. (1974), The Einstein Decade (1905-1915), New York, Academic Press.

MERleau-Ponty J. (1974), Leçons sur la genèse des théories physiques, Paris, Vrin.

POINCARÉ H. [1902] (1968), La Science et l'hypothèse, Paris, Flammarion.

PoINCARÉ H. [1905] (1970), La Valeur de la science, Paris, Flammarion.

SCHEIBE E. (1978), "On the structure of physical Theories", Acta Philosophica Fennica, 30, The Logic and Epistemology of Scientific Change, p. 205-224.

SNEED J. D. (1971), The Logical Structure of Mathematical Physics, Dordrecht, Reidel.

Trautman A. (1966a), "The General Theory of Relativity», Soviet Physics Uspekhi, 9, 3, p. 319-339.

Trautman A. (1966b), "Comparison of Newtonian and Relativistic Theories of Space-Time", Perspectives in Geometry and Relativity, B. Hoffman (éd.), Bloomington, Indiana University Press, p. 413-425.

Trautman A. (1973), "Theory of Gravitation", in The Physicist's Conception of Nature, J. Mehra (éd.), Dordrecht, Reidel. 